\title{
Time-Varying High-Gain Trajectory Linearization Observer Design
}

\author{
Rui Huang and J. Jim Zhu
}

\begin{abstract}
In this paper, we extend the previous results on trajectory linearization observer (TLO) for SISO to MIMO nonlinear time-varying (NLTV) systems, and extend the highgain observer theory for linear time-invariant (LTI) observer error dynamics to linear time-varying (LTV) observer error dynamics, using the (time-varying) PD-eigenvalue assignment method. In addition, the time-varying high gain TLO alleviates the slowly varying restriction, and relaxes the restriction of existing time-invariant nonlinear high gain observer to affine nonlinear systems to allow for any linearizable NLTV system.
\end{abstract}

Key Word: MIMO, TLO, nonlinear systems, nonlinear observer, trajectory linearization, linear time-varying

\section{INTRODUCTION}

Most nonlinear control design techniques are developed for full state feedback, assuming that measurements of all states are available. However, in practical control problems, usually not all states are measured due to economical or technical reasons. Thus, nonlinear observer design techniques are needed to perform output feedback $[1,2]$.

An effective approach to nonlinear observer design is to reduce the observer error dynamics (OED) to linear dynamics. There are essentially two classes of methods for the reduction: (i) transforming the OED to a topologically equivalent linear system via a diffeomorphism or homeomorphism, and (ii) approximating the NLTV OED in the neighborhood of the null equilibrium by using the linear term of its Taylor expansion.

The Class (i) approaches are typified ranging from the pioneering work of Krener and Isidori [3] to the most recent advancements by Xiao [4] and Kravaris et. al. [5] (see also references therein). The linear OED are obtained either by a homeomorphism, as typified by [4] or a diffeomorphism, as typified by [5], whereby the OED is stabilized in the transformed coordinate, and stability in the original coordinate is ensured by the boundedness of the transformations. In theory, this class of linearization is exact. In practice, finding the transforms may necessitate approximations, and the implementation entails output injection, which is subject to sensor dynamics, precision and noise. These practical artifacts introduce regular and singular perturbations into the implemented design. While in principle this class of methods are applicable to NLTV systems, the present results are confined to time-invariant nonlinear systems.

Manuscript received Sep 21, 2008

Rui Huang (Email: ruihuang@bobcat.ent.ohiou.edu) and J. Jim Zhu (corresponding author. Phone: (740)597-1506; Fax: (740)593-0007; Email: zhuj@ohio.edu) are with the School of Electrical Engineering and Computer Science, Ohio University, Athens, Ohio 45701, USA
The Class (ii) methods are more classical, whereby linear approximation of the OED is taken either along the (yet to be estimated) state trajectory, as typified by extended/linearized (EKF/LKF) Kalman filter type techniques $[6,7]$, or at discrete points either along the state trajectory or in the operating envelope, as typified by the classical work of Baumann and W. J. Rugh [8]. Note that trajectory linearization results in LTV OED even if the nonlinear plant is time-invariant. The premise of this class of approach is that the observer errors are sufficiently small so that the neglected nonlinearity terms can be treated as a vanishing regular perturbation. Exponential stability of the linearized OED is retained for the nonlinear system due to the vanishing nature of the neglected nonlinearity. Additional regular perturbations are introduced from the use of alternative nominal trajectories for trajectory linearization, since the nominal trajectory is yet to be estimated, or from discrete-point linearization. Stabilization of the LTV OED is achieved either by Kalman filter techniques, where process and sensor noise models need to be assumed even if the design is only for a deterministic system, or by gain-scheduling method, which is subject to the slowly varying assumption on the state trajectory and NLTV plant parameters. Perhaps the single most desirable feature of Class (ii) approach is its wide applicability to any NLTV system defined by a differentiable vector field.

In order to accommodate the unavoidable regular and singular perturbations, disturbances and sensor noise that enter the observer dynamics, regardless of which class of design approach, the linearized OED should be designed for exponential stability, albeit locally. The stabilized linearized observer dynamics are almost invariably synthesized to LTI dynamics using eigenvalue (pole) placement for Class (i) methods and for gain-scheduling Class (ii) methods. The stabilized OED for EKF/LKF type of methods are LTV in nature, which ensures that the error covariance is minimized along the (time-varying) trajectory and in the presence of NLTV plant parameter variations. It is noted that, although widely used in practice for decades, the optimality of Kalman filter for linear systems and the principle of certainty equivalence do not carry over to NLTV systems. Therefore, these methods are more for stabilization than optimality. However, the stability of EKF was not proven until very recently (Krener [9], 2003) for uniformly observable Class $\mathcal{C}^{2}$ systems.

Employing an observer for output feedback control, introduces a singular perturbation into the full state feedback control law design. High-gain observer design techniques $[2,10,11]$ have been introduced for LTI OED to reduce the 
perturbation in order to recover the performance of full-state feedback. High-gain observer can also reduce regular perturbation to the observer dynamics due to plant modeling errors. High-gain observers may cause a peaking phenomenon, which can be dealt with using control saturation [12].

The trajectory linearization observer (TLO) method presented in [13] is a Class (ii) technique for NLTV systems, which had broad applicability. In this paper, we extend the results for SISO TLO [13] to MIMO NLTV systems, and extend the high-gain observer theory for LTI OED to LTV OED. Both extensions are nontrivial and rely heavily on the unique (time-varying) PD-eigenvalue assignment stabilization method [14]-[16] for the LTV OED. TLO can be viewed as an extension of gain-scheduled observer that is designed and exponentially stabilized along the state trajectory, rather than at discrete operating points, thereby alleviating the slowly varying restriction on state trajectory and NLTV system parameters. Comparing to EKF/LKF type of observers, the PD-eigenvalue assignment method provides a more intuitive way that not only guarantees exponential stability of the OED, but also allows timevarying observer gain (bandwidth) to be employed for realtime adaptation or tradeoff between steady-state observer errors, peaking and robustness to modeling errors.

The PD-spectral [14]-[16] theory, regular perturbation, singular perturbation and nonlinear high-gain observer theories are applied to analyze the stability and robustness of the TLO with time-varying observer gains. The main result shows that, using high-gain (high-bandwidth), the TLO based output feedback recovers the state feedback performance, even for time-varying observer gains (bandwidth). Moreover, the time-varying high-gain TLO relaxes the existing time-invariant nonlinear high gain observer's restriction to affine nonlinear systems to allow any linearizable NLTV system. A MIMO high-gain TLO design example with hardware-in-loop simulation test for a 3 Degrees-of-Freedom (3DOF) differential thrust control testbed named "Quanser UFO" is presented in [17]. A simulation comparison study of the proposed TLO with EKF for an unstable, nonminimum phase, stiff nonlinear benchmark model is presented in [18].

The paper is structured as follows. In section II, trajectory linearization control (TLC) and existing results of TLO are briefly reviewed, and the TLO method is extended to MIMO systems. In section III existing LTI high-gain observer theory is extended for LTV OED. A summary of the paper is presented in section IV.

\section{MIMO Trajectory Linearization Observer}

Consider output tracking by a nonlinear time-varying dynamic system

$$
\dot{\xi}(t)=f(\xi(t), \mu(t), t), \quad \eta(t)=h(\xi(t), \mu(t), t)
$$

where $\xi(t) \in \mathbb{R}^{n}, \mu(t) \in \mathbb{R}^{l}, \eta(t) \in \mathbb{R}^{m}$ are the state, input and output respectively, and the mappings $f(\cdot, \cdot, \cdot): \mathbb{R}^{n} \times$ $\mathbb{R}^{l} \times \mathbb{R} \rightarrow \mathbb{R}^{n}$ and $h(\cdot, \cdot): \mathbb{R}^{n} \times \mathbb{R}^{l} \rightarrow \mathbb{R}^{m}$ are bounded and locally Lipschitz. Let $\bar{\xi}(t), \bar{\eta}(t), \bar{\mu}(t)$ be the nominal state, nominal output trajectories and nominal control satisfying

$$
\dot{\bar{\xi}}(t)=f(\bar{\xi}(t), \bar{\mu}(t), t) \quad \bar{\eta}(t)=h(\bar{\xi}(t), \bar{\mu}(t), t)
$$

and define tracking errors by

$$
\tilde{\xi}(t)=\xi(t)-\bar{\xi}(t) \quad \tilde{\eta}(t)=\eta(t)-\bar{\eta}(t) \quad \tilde{\mu}(t)=\mu(t)-\bar{\mu}(t)
$$

Then the tracking error dynamics are governed by

$$
\begin{aligned}
\dot{\tilde{\xi}}(t) & =f(\bar{\xi}(t)+\tilde{\xi}(t), \bar{\mu}(t)+\tilde{\mu}(t), t)-f(\bar{\xi}(t), \bar{\mu}(t), t) \\
& =F(\tilde{\xi}(t), \tilde{\mu}(t), \bar{\xi}(t), \bar{\mu}(t), t) \\
\tilde{\eta}(t) & =h(\bar{\xi}(t)+\tilde{\xi}(t), \bar{\mu}(t)+\tilde{\mu}(t), t)-h(\bar{\xi}(t), \bar{\mu}(t), t) \\
& =H(\tilde{\xi}(t), \tilde{\mu}(t), \bar{\xi}(t), \bar{\mu}(t), t)
\end{aligned}
$$

It is noted that the nonlinear tracking error dynamics and its linearization are time-varying for time-varying nominal trajectories, even if the nonlinear plant (1) is time-invariant. This fact calls for intrinsically time-varying controller and observer design methods, such as the TLC and TLO.

\section{A. Trajectory Linearization Control}

As shown in Figure 1, state trajectory linearization control consists of two parts: a dynamic inverse I/O mapping of the plant to compute the nominal control function $\bar{\mu}(t)$ for any given nominal output trajectory $\bar{\eta}(t)$, and a tracking error stabilizing control law $\tilde{\mu}(\tilde{\xi}, \bar{\xi})$ to account for modeling simplifications and uncertainties, and disturbances.

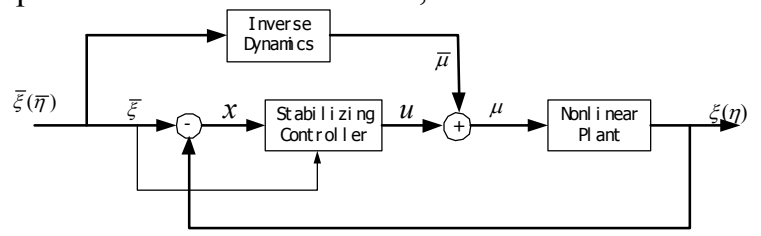

Figure 1 State feedback TLC Configuration

The nominal control $\bar{\mu}$ can be designed using a pseudo nonlinear dynamic inversion technique [19]. With the assumption that the tracking errors are small by performance requirement, the tracking error dynamics can be linearized along the nominal trajectory as

$$
\dot{x}=A_{c}(t) x+B_{c}(t) u \quad y=C_{c}(t) x+D_{c}(t) u
$$

where $x, u, y$ are the linear approximations to $\xi, \mu, \eta$ respectively, and

$$
\begin{aligned}
A_{c}(t) & =A(\bar{\xi}(t), \bar{\mu}(t))=\left.\frac{\partial}{\partial \xi} f(\xi, \mu, t)\right|_{\bar{\xi}, \bar{\mu}} \\
B_{c}(t)=B(\bar{\xi}(t), \bar{\mu}(t)) & =\left.\frac{\partial}{\partial \mu} f(\xi, \mu, t)\right|_{\bar{\xi}, \bar{\mu}} \\
C_{c}(t)=C(\bar{\xi}(t), \bar{\mu}(t)) & =\left.\frac{\partial}{\partial \xi} h(\xi, \mu, t)\right|_{\bar{\xi}, \bar{\mu}} \\
D_{c}(t)=D(\bar{\xi}(t), \bar{\mu}(t)) & =\left.\frac{\partial}{\partial \mu} h(\xi, \mu, t)\right|_{\bar{\xi}, \bar{\mu}}
\end{aligned}
$$

which can be stabilized using a LTV control law $u=K(t) x$, with the assumption that the system is strongly controllable [20]. The LTV gain $K(t)$ can be computed symbolically using the PD-eigenstructure assignment approach [19, 14].

\section{B. TLO Design procedure}

The TLO design method is inspired by and based on the TLC algorithm. The output tracking error TLC is shown in 
Fig. $2^{1}$, where the state error $\tilde{\xi}(t)$ is estimated from the input $\mu(t)$ and the output error $\tilde{\eta}(t)$. The output error TLO differs from the state TLO [13] in that the latter is derived in the state coordinate, whereas the former is in the tracking error coordinate. However, the observer gain obtained in both configurations can be proven to be identical [18].

Given the nonlinear system (1), design a nonlinear tracking error observer as

$$
\begin{aligned}
& \dot{\tilde{\tilde{\xi}}}=F(\widehat{\tilde{\xi}}(t), \tilde{\mu}(t), \bar{\xi}(t), \bar{\mu}(t))+K_{o}(t)(\widehat{\tilde{\eta}}-\tilde{\eta}) \\
& \widehat{\tilde{\eta}}=H(\widehat{\tilde{\xi}}(t), \tilde{\mu}(t), \bar{\xi}(t), \bar{\mu}(t), t)
\end{aligned}
$$

where $F(,$,$) and H(, \cdot)$ are defined in $(3), \widehat{\tilde{\xi}}(t)$ and $\widehat{\tilde{\eta}}(t)$ are the estimated state error and output error vectors, respectively, and $K_{o}(t)$ is the observer gain to be designed using the trajectory linearization method for exponential stability.

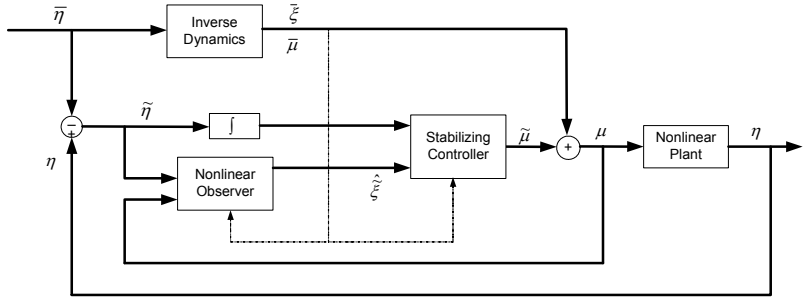

Figure 2 Output feedback TLC with tracking error observer

Step (a) Linearization along the nominal trajectory

Linearize (5) along the nominal trajectory $\tilde{\xi}(t)$ to obtain

$$
\begin{aligned}
& \dot{\hat{x}}=A_{o}(t) \widehat{x}+K_{o}(t) \widehat{y}=\left(A_{o}(t)+K_{o}(t) C_{o}(t)\right) \widehat{x} \\
& \widehat{y}=C_{o}(t) \widehat{x}
\end{aligned}
$$

where $\widehat{x} \approx \widehat{\tilde{\xi}}-\tilde{\xi}$ is the linearized observer error, $\hat{y}=\hat{\tilde{\eta}}-\tilde{\eta}$, and

$$
\begin{aligned}
& A_{o}=A(\tilde{\xi}, \mu)=\left.\frac{\partial}{\partial \widehat{\tilde{\xi}}} F\right|_{\tilde{\xi}}=\left.\frac{\partial}{\partial \widehat{\tilde{\xi}}} f(\bar{\xi}+\widehat{\tilde{\xi}}, \bar{\mu}+\tilde{\mu})\right|_{\tilde{\xi}} \\
& C_{o}=C(\tilde{\xi}, \mu)=\left.\frac{\partial}{\partial \widehat{\tilde{\xi}}} H\right|_{\tilde{\xi}}=\left.\frac{\partial}{\partial \widehat{\tilde{\xi}}} h(\bar{\xi}+\widehat{\tilde{\xi}}, \bar{\mu}+\tilde{\mu})\right|_{\tilde{\xi}}
\end{aligned}
$$

\section{Step (b) PD-eigenvalue assignment for MIMO LTV system}

It is noted that the PD-eigenvalues are not the frozen-time eigenvalues, the latter have been proven to be neither a sufficient [21] nor a necessary [22] condition for stability. The observer PD-eigenvalue assignment consists of three steps: (i) transform the linearized OED into the LTV observer canonical form, (ii) assign the desired PDeigenvalues using the synthesis formula given in [15][16], and (iii) perform the inverse transformation to obtain the observer gain matrix. The procedure for SISO system is presented in [13]. In this paper, we extend the algorithm to MIMO systems. The procedure is described in detail below.

(i) Observer canonical form transformation

Controller canonical form transformation for LTI MIMO systems is presented in [23].Silverman [24] developed the transformation to controller canonical form for SISO LTV systems, which was extended to observer canonical form by Bestle [25]. Wolovich [26] first developed controller canonical form transformation for MIMO LTV systems, and Seal [27] improved the procedure and notations. However,

\footnotetext{
${ }^{1}$ The integral feedback shown in Fig. 2 is not considered in this paper.
}

the transformation for LTV MIMO systems is still difficult to follow. Inspired by [23]-[27], we use time-varying Lie derivatives to reconstruct the algorithm and extend it to MIMO observer canonical form based on the duality of controllability and observability.

Consider the $n$-th order LTV system,

$$
\dot{x}=A(t) x \quad y=C(t) x
$$

where the pair $\left\{A(\cdot): \mathbb{R} \rightarrow \mathbb{R}^{n \times n}, C(\cdot): \mathbb{R} \rightarrow \mathbb{R}^{m \times n}\right\}$ is said to be strongly observable if and only if its observability matrix $Q(t)$ satisfies $\left|\operatorname{det}\left[Q_{o}^{T}(t) Q_{o}(t)\right]\right| \geq c, \forall t \geq t_{0}$ where

$$
Q(t)=\left[\begin{array}{llll}
C(t)^{T} & (\mathcal{L} C(t))^{T} & \cdots & \left(\mathcal{L}^{n-1} C(t)\right)^{T}
\end{array}\right]
$$

with the differential operator $\mathcal{L}$ defined as $\mathcal{L} C(t)=$ $\dot{C}(t)+C(t) A(t)$. Assume LTV system (8) is strongly observable and has fixed lexicographic basis. Define $p_{i}, i=1$, $\cdots, m$ as the observability index of the system. We have $\sum_{i=1}^{m} p_{i}=n$. Also define $d_{i}=\sum_{k=1}^{i} p_{k}$. Then (8) can be transformed into the MIMO time-varying observer canonical form

where

$$
\begin{aligned}
& \dot{z}=A_{z}(t) z=A_{\text {can }}(t) z, \quad z\left(t_{0}\right)=z_{0} \\
& y=C_{z} z=R(t) C_{\text {can }}(t) z
\end{aligned}
$$

$$
\begin{gathered}
A_{\text {can }}(t)=\left[\begin{array}{cccc}
A_{11}(t) & A_{12}(t) & \cdots & A_{1 m}(t) \\
A_{21}(t) & A_{22}(t) & \cdots & A_{2 m}(t) \\
\vdots & \vdots & \ddots & \vdots \\
A_{m 1}(t) & A_{m 2}(t) & \cdots & A_{m m}(t)
\end{array}\right] \\
\left.C_{\text {can }}(t)=\text { block diagonal }\left\{\begin{array}{llll}
0 & \cdots & 0 & 1
\end{array}\right]_{1 \times p_{i}}\right\}
\end{gathered}
$$

where

$$
\begin{aligned}
& A_{i i}=\left[\begin{array}{cccc}
0 & \cdots & 0 & \alpha_{d_{i-1}+1, i}(t) \\
1 & \cdots & 0 & \alpha_{d_{i-1}+2, i}(t) \\
\vdots & \ddots & \vdots & \vdots \\
0 & \cdots & 1 & \alpha_{d_{i}, i}(t)
\end{array}\right]_{p_{i} \times p_{i}} \quad i=1, \cdots, m \\
& A_{k i}=\left[\begin{array}{cccc}
0 & \cdots & 0 & \alpha_{d_{k-1}+1, i}(t) \\
0 & \cdots & 0 & \alpha_{d_{k-1}+2, i}(t) \\
\vdots & \ddots & \vdots & \vdots \\
0 & \cdots & 0 & \alpha_{d_{k}, i}(t)
\end{array}\right]_{p_{k} \times p_{i}} \begin{array}{l}
i=1, \cdots, m \\
k \neq i
\end{array}
\end{aligned}
$$

and $R(t)$ is an $m \times m$ invertible upper-triangular matrix with unity diagonal entries. Define the following operators and interim variables that will be used in the sequel

$$
\begin{gathered}
\tilde{\mathcal{L}} q(t)=-\dot{q}(t)+A(t) q(t) \\
\tilde{\Gamma}_{i}(q(t))=\left[\begin{array}{ccc}
q(t), & \tilde{\mathcal{L}} q(t), \quad \cdots & \tilde{\mathcal{L}}^{i-1} q(t)
\end{array}\right] \\
\Gamma_{i}(c(t))=\left[\begin{array}{c}
c(t) \\
\mathcal{L} c(t) \\
\vdots \\
\mathcal{L}^{i-1} c(t)
\end{array}\right] \quad Q_{0}(t)=\left[\begin{array}{c}
\Gamma_{p_{1}}\left(c_{1}(t)\right) \\
\Gamma_{p_{2}}\left(c_{2}(t)\right) \\
\vdots \\
\Gamma_{p_{m}}\left(c_{m}(t)\right)
\end{array}\right]
\end{gathered}
$$

where $c_{i}(t), i=1, \cdots, m$ are the $i$ th row-vectors of $C(t)$. Then the time-varying coordinate transformation

$$
z=T(t) x
$$

that transforms (8) into the observer canonical form (9) is given in terms of $T^{-1}(t)$ by

$$
T^{-1}(t)=\left[\tilde{\Gamma}_{p_{1}}\left(q_{1}\right)\left|\tilde{\Gamma}_{p_{2}}\left(q_{2}\right)\right| \cdots \mid \tilde{\Gamma}_{p_{m}}\left(q_{m}\right)\right]
$$

where $q_{i}(t)=Q_{0}^{-1}(t) e_{d_{i}}, i=1, \cdots, m$, is the $d_{i}^{\text {th }}$ column vector of $Q_{0}^{-1}(t)$ and $e_{k}$ is the $k^{\text {th }}$ standard basis vector for $\mathbb{R}^{n}$. Apply (10) to the linearized observer error (6) with 
$\widehat{x}=T^{-1}(t) z$ to obtain the observer canonical form

$$
\begin{aligned}
& \dot{z}=\left(A_{z}(t)+K_{z}(t) C_{z}(t)\right) z=\tilde{A}_{z}(t) z \\
& y=C_{z}(t) z
\end{aligned}
$$

where

$$
\begin{aligned}
& A_{z}(t)=T(t) A_{o}(t) T^{-1}(t)+\dot{T}(t) T^{-1}(t) \\
& C_{z}(t)=C_{o}(t) T^{-1}(t), K_{z}(t)=T(t) K_{o}(t)
\end{aligned}
$$

\section{(ii) PD-eigenvalue assignment [15][16]}

Denote $K_{z}(t)=\left[k_{j, i}(t)\right]_{j=1, \cdots, n, i=1, \cdots, m}$, where $k_{j, i}(\cdot)$ are sufficiently differentiable. Then the Right Polynomial Differential Operators (RPDO) [15] associated with the $i^{\text {th }}$ subsystem of $\tilde{A}_{z}$ is given as

$$
\begin{aligned}
D_{\alpha_{i}}= & \delta^{n}-\delta^{n-1}\left(\alpha_{d_{i-1}+p_{i}, i}(t)+k_{d_{i-1}+p_{i}, i}(t)\right) \\
& -\cdots-\delta\left(\alpha_{d_{i-1}+2, i}(t)+k_{d_{i-1}+2, i}(t)\right) \\
& -\left(\alpha_{d_{i-1}+1, i}(t)+k_{d_{i-1}+1, i}(t)\right)
\end{aligned}
$$

where $\delta=d / d t$ is the derivative operator. Place the closed loop PD-spectrum $\left\{\rho_{i}(t)\right\}_{i=1}^{n}$ according to the tracking performance requirement and the singular perturbation principle. Refer to [15][13] for the PD-spectral synthesis formula for given PD-spectrum $\left.\left\{\rho_{i}(t)\right\}_{i=1}^{n}\right)$. Note that the coefficients obtained from the synthesis formula are for LPDO, thus need to be transformed into RPDO to get $\left\{\beta_{p_{i}, i}(t)\right\}^{k=1, \cdots, p_{i}}$. Denote the RPDO associated with the PDspectrum as

$$
D_{\beta_{i}}=\delta^{n}+\delta^{n-1} \beta_{p_{i}, i}(t)+\cdots+\delta \beta_{2, i}(t)+\beta_{1, i}(t)
$$

The coupled terms between different subsystems will be canceled by the observer gain, which yields the observer gain under the canonical coordinate as

$$
\begin{aligned}
& k_{d_{s-1}+k, i}= \begin{cases}\beta_{k, i}-\alpha_{d_{s-1}+k, i} & s=i \\
-\alpha_{d_{s-1}+k, i} & s \neq i\end{cases} \\
& i=1, \cdots, m ; \quad k=1, \cdots, p_{s} ; \quad s=1, \cdots, m ;
\end{aligned}
$$

(iii) Computing the observer gain

Taking the inverse canonical transformation of (10), the observer gain is given by

\section{Step (c) Implementation}

$$
K_{o}(t)=T^{-1}(t) K_{z}(t)
$$

A problem in implementation is that $K_{o}(t)$ is a function of $\tilde{\xi}(t)$, which is yet to be estimated. A solution that is similar to the one proposed in [13] is to use $K_{o}(0, \mu(t))$ as a substitution for $K_{o}(\tilde{\xi}(t), \mu(t))$ to implement the observer. A theory [18] was derived in the same manner as the one in [13] to prove that the stability of the overall system is retained with the replacement.

\section{TIME-VARYING HIGH GAIN TLO}

In the preceding section, the TLO is designed assuming no modeling error. However, in practice, modeling errors are unavoidable and can be nontrivial, which enter the OED as a regular perturbation, and may degrade the performance or even destabilize the overall system. In this section, a nonlinear time-varying bandwidth (TVB) observer is developed as an effective means for handling modeling errors and more. The proposed approach is inspired by the nonlinear high-gain observer developed by Khalil in [2], in which high-gain and control saturation are employed in output feedback controller to effectively recover the performance under state feedback controller. However, the Khalil high- gain observer (HGO) suffers from the following two main restrictions. First, it is only applicable to nonlinear time invariant systems in the affine form such that feedback linearization can be performed to obtain the time invariant normal form. Moreover, the Khalil HGO is restricted to nonlinear systems with relative degree $n$ and with all the modeling errors satisfying the corresponding matching conditions. For systems with relative degree less than $n$, applying feedback linearization renders the $n-r$ internal states unobservable. The second limitation of Khalil HGO is that it allows only constant observer gain, while time-varying gain is highly desirable in the nonlinear observer design. For instance, in coping with the peaking phenomenon in HGO, control saturation is used, which is actually nothing but a time-varying gain scheme. Thus, better performance may be achieved by effectively varying the controller or observer gain in some optimal way. The proposed TVB TLO design, on the other hand, only requires linearizable model, which is more general than the affine form. Furthermore, timevarying eigenstructure assignment is enabled by virtue of the PD-spectrum theory adopted in the TVB TLO design, which allows the observer gain to vary in coping with the performance and robustness tradeoffs. Some restrictions in Khalil HGO are not relaxed in this paper, such as requirement of relative degree $n$ and matching condition, which will be left as open topics for future research that are very likely solvable using the TLO approach.

\section{A. Motivation and Solution}

Two technical difficulties arise when attempting to extend the high-gain observer theory to the time-varying case: one is how to scale the observer gains without jeopardizing the stability of the time-varying system; the other is how to relate the steady state error with the scaling factor in timevarying systems. These two problems will be solved in the following sections using the PD-spectral theory such that the high gain theory is extended to the time-varying TLO.

Consider NLTV tracking error dynamics (3). We use a two-step approach to design the output feedback error regulating controller. First, a state feedback controller is designed to exponentially stabilize the origin assuming all state variables are measured. Then, a time-varying high-gain observer is used to estimate $x$ from the output $y$. The state feedback control law is

$$
\tilde{\mu}=K(t) \tilde{\xi}
$$

For convenience, we write the closed-loop system under state feedback as

$$
\begin{aligned}
& \dot{\tilde{\xi}}=F(\tilde{\xi}(t), K(t) \tilde{\xi}(t), t, \bar{\xi}(t), \bar{\mu}(t)) \\
& \tilde{\eta}=H(\tilde{\xi}(t), K(t) \tilde{\xi}(t), t, \bar{\xi}(t), \bar{\mu}(t))
\end{aligned}
$$

The output feedback control law is implemented as

$$
\tilde{\mu}=K(t) \widehat{\tilde{\xi}}
$$

where $\widehat{\tilde{\xi}}$ is generated by the time-varying high-gain observer

$$
\begin{aligned}
& \dot{\tilde{\tilde{\xi}}}=F(\widehat{\tilde{\xi}}(t), \tilde{\mu}(t), \bar{\xi}(t), \bar{\mu}(t), t)+K_{o_{H}}(\cdot)(\widehat{\tilde{\eta}}-\tilde{\eta}) \\
& \widehat{\tilde{\eta}}=H(\widehat{\tilde{\xi}}(t), \tilde{\mu}(t), \bar{\xi}(t), \bar{\mu}(t))
\end{aligned}
$$

Linearize the observer dynamics along its nominal trajectory $\tilde{\xi}$, and obtain the OED as 


$$
\begin{aligned}
\dot{\hat{\tilde{x}}}= & \left(A_{o}(t)+K_{o_{H}}(\cdot) C_{o}(t)\right) \widehat{\widetilde{x}} \\
& +B_{o}(t)\left(\delta_{v}(\tilde{\xi}, \widehat{\widetilde{x}}, \tilde{\mu}, t, \bar{\xi}(t), \bar{\mu}(t))+\delta_{n v}(\tilde{\xi}, \widehat{\widetilde{x}}, \tilde{\mu}, t)\right) \\
\widehat{\widetilde{y}}= & C_{o}(t) \widehat{\widehat{x}}
\end{aligned}
$$

where $\widehat{\widehat{x}} \approx \widehat{\tilde{\xi}}-\tilde{\xi}, \widehat{\hat{y}} \approx \widehat{\tilde{\eta}}-\tilde{\eta}$ are the linear approximations to $\tilde{\widehat{\xi}}$ and $\tilde{\tilde{\eta}}$ respectively, and $A_{o}$ and $C_{o}$ are obtained in equation (7), $B_{o}(t)$ is given as

$$
B_{o}=B(\tilde{\xi}, \mu)=\left.\frac{\partial}{\partial \mu} F\right|_{(\tilde{\xi}, \mu)}
$$

and $\delta_{v}(\cdot)$ is a vanishing perturbation consisting of linearization error, vanishing part of the modeling error and implementation error, as discussed in [13] and $\delta_{n v}(\cdot)$ is a nonvanishing perturbation which accounts for the non-vanishing part of the modeling error. Notice here, we assume that the perturbations satisfy the matching condition ${ }^{2}$.

Assumption 1 Assume $\delta_{v}(\tilde{\xi}, \widehat{\tilde{x}}, \tilde{\mu}, t, \overline{\boldsymbol{\xi}}(t), \overline{\boldsymbol{\mu}}(t))$ and $\delta_{n v}(\tilde{\xi}, \widehat{\tilde{x}}, \tilde{\mu}, t)$ are Lipschitz in all its arguments except $t$ over the domain of interest, uniform in t and satisfy

$$
\begin{aligned}
& \left\|\delta_{v}(\tilde{\xi}, \widehat{\tilde{x}}, \tilde{\mu}, t, \overline{\boldsymbol{\xi}}(t), \overline{\boldsymbol{\mu}}(t))\right\| \leq L_{1} \quad \forall t \geq 0 \\
& \left\|\delta_{n v}(\tilde{\xi}, \widehat{\tilde{x}}, \tilde{\mu}, t)\right\| \leq L_{2}
\end{aligned}
$$

where $L_{1}$ and $L_{2}$ are positive constants.

Equation (19) can be transformed into LTV observer canonical form by a Lyapunov coordinate transformation $\tilde{w}=T_{1}(t) \widehat{\widehat{x}}$, yielding

$$
\dot{\tilde{w}}=\left(A_{w}(t)+K_{o_{w}}(t) C_{w}(t)\right) \tilde{w}+B_{w}(t) \Delta
$$

where $A_{w}, K_{\mathrm{o}_{w}}$ and $C_{w}$ are obtained by equation (11), and

$$
\Delta=\left(\delta_{v}\left(\tilde{\xi}, T_{1}^{-1}(t) \tilde{w}, \tilde{\mu}, t, \overline{\boldsymbol{\xi}}(t), \overline{\boldsymbol{\mu}}(t)\right)+\delta_{n v}\left(\tilde{\xi}, T_{1}^{-1}(t) \tilde{w}, \tilde{\mu}, t\right)\right)
$$

and $B_{w}(t)=T_{1}^{-1} B_{o}(t)$. The observer gain $K_{o_{w}}(t)$ is obtained as in (14). Only for high gain observer, the PD-spectrum is chosen as $\Upsilon_{H_{i}}=\left\{\rho_{H_{k}}(t)=\rho_{k}(t) / \epsilon\right\}_{k=1}^{p_{i}}, i=1, \cdots, m$, where $\Upsilon_{i}=\left\{\rho_{k}(t)\right\}_{k=1}^{p_{i}}, i=1, \cdots, m$, is the PD-spectrum chosen for TLO introduced in the preceding section, hereafter called the reference TLO, and $0<\epsilon<1$ is a positive constant.

Next, we present a convenient and practical scheme to scale the observer gains for time-varying systems such that the stability of the systems is retained and the scaling factor $\frac{1}{\epsilon}$ possesses some physical meanings.

In practical applications, it is convenient ${ }^{3}$ to choose the PD-spectrum as $\Upsilon_{i}=\left\{\rho_{k}(t)=\bar{\rho}_{k} \omega(t)\right\}_{k=1}^{p_{i}}$. Thus,

$$
\Upsilon_{H_{i}}=\left\{\rho_{H_{k}}(t)=\bar{\rho}_{k} \cdot \omega_{H}(t)\right\}_{k=1}^{p_{i}}
$$

where, $\omega_{H}(t)=\omega(t) / \epsilon$. The PD-modal matrix $V_{p_{i}}\left(\Upsilon_{i}\right)$ for the reference TLO can be written as

$$
V_{p_{i}}\left(\Upsilon_{i}\right)=\widehat{V}_{p_{i}}(\omega) \bar{V}_{p_{i}}(\bar{\rho})
$$

where

$$
\overline{\boldsymbol{V}}_{p_{i}}(\bar{\rho})=\left[\begin{array}{cccc}
1 & 1 & \cdots & 1 \\
\bar{\rho}_{1} & \bar{\rho}_{2} & \cdots & \bar{\rho}_{p_{i}} \\
\vdots & \vdots & \ddots & \vdots \\
\bar{\rho}_{1}^{p_{i}-1} & \bar{\rho}_{2}^{p_{i}-1} & \cdots & \bar{\rho}_{p_{i}}^{p_{i}-1}
\end{array}\right]
$$

\footnotetext{
${ }^{2}$ The assumption of the matching condition weakens the strength and the applicability of the proposed theory. While it is needed for the proof of the theorem in the next section, it is not essential. This limitation is a consequence of the method of proof inhered from the time-invariant HGO theory [12]. Future work is needed to relax the assumption.

${ }^{3}$ When the PD-spectrum is chosen as the product of constant nominal eigenvalues $\bar{\rho}_{k}$ and a time-varying bandwidth $\omega(t)$, the synthesis formula becomes much simpler.
}

is the Vandermonde matrix associated with $\left\{\bar{\rho}_{i}\right\}_{i=1}^{p_{i}}$, and

$$
\widehat{\boldsymbol{V}}_{p_{i}}(\omega)=\left[\begin{array}{cccc}
1 & 0 & \cdots & 0 \\
0 & \widehat{v}_{22}(t) & \ddots & \vdots \\
\vdots & \vdots & \ddots & 0 \\
0 & \widehat{v}_{n 2}(t) & \cdots & \widehat{v}_{n n}(t)
\end{array}\right]
$$

where

$$
\widehat{v}_{i j}(t)=\dot{\widehat{v}}_{i-1, j}(t)+\omega(t) \widehat{v}_{i-1, j-1}, \quad 2 \leq j \leq i \leq n
$$

For example the $\widehat{\boldsymbol{V}}_{p_{i}}(\omega)$ of a fourth order system is given as

$$
\widehat{\boldsymbol{V}}_{p_{i}}(\omega)=\left[\begin{array}{cccc}
1 & 0 & 0 & 0 \\
0 & \omega(t) & 0 & 0 \\
0 & \dot{\omega}(t) & \omega^{2}(t) & 0 \\
0 & \ddot{\omega}(t) & 3 \omega(t) \dot{\omega}(t) & \omega^{3}(t)
\end{array}\right]
$$

The PD-modal matrix $V_{p_{i}}\left(\Upsilon_{H_{i}}\right)$ for high-gain TLO can be written as

$$
V_{p_{i}}\left(\Upsilon_{H_{i}}\right)=\widehat{V}_{p_{i}}\left(\omega_{H}\right) \bar{V}_{p_{i}}(\bar{\rho})
$$

where

$$
\widehat{\boldsymbol{V}}_{p_{i}}\left(\omega_{H}\right)=\left[\begin{array}{cccc}
1 & 0 & \cdots & 0 \\
0 & \widehat{v}_{22}(t) & \ddots & \vdots \\
\vdots & \vdots & \ddots & 0 \\
0 & \widehat{v}_{n 2}(t) & \cdots & \widehat{v}_{n n}(t)
\end{array}\right]
$$

where $\widehat{v}_{i j}(t)=\dot{\widehat{v}}_{i-1, j}(t)+\omega_{H}(t) \widehat{v}_{i-1, j-1}, \quad 2 \leq j \leq i \leq n$. For example the $\widehat{\boldsymbol{V}}_{p_{i}}\left(\omega_{H}\right)$ of a 4 th-order system is given as

$$
\widehat{\boldsymbol{V}}_{p_{i}}\left(\omega_{H}\right)=\left[\begin{array}{cccc}
1 & 0 & 0 & 0 \\
0 & \omega(t) \frac{1}{\epsilon} & 0 & 0 \\
0 & \dot{\omega}(t) \frac{1}{\epsilon} & \omega^{2}(t) \frac{1}{\epsilon^{2}} & 0 \\
0 & \ddot{\omega}(t) \frac{1}{\epsilon} & 3 \omega(t) \dot{\omega}(t) \frac{1}{\epsilon^{2}} & \omega^{3}(t) \frac{1}{\epsilon^{3}}
\end{array}\right]
$$

It is noted that both the TVB reference TLO dynamics and the high gain scaling $\epsilon^{-1}$ allow the observer dynamics to be time-scaled with a constant damping. Moreover, the relationship (23) facilitates the extension of the HGO theory to LTV observer dynamics.

\section{B. Theorem and Proof}

In this subsection, we develop a theorem which shows that the steady state error of the overall closed-loop system under output feedback control caused by modeling error can be made arbitrarily small by scaling up the time-varying bandwidth of the observer dynamics.

The OED (19) can be transformed into LTV observability canonical form by a Lyapunov coordinate transformation $\tilde{z}=T_{2}(t) \widehat{\tilde{x}}$, yielding

$$
\dot{\tilde{z}}=\left(A_{z}(t)+K_{o_{z}}(t) C_{z}(t)\right) \tilde{z}+B_{z}(t) \Delta
$$

where $A_{z}, K_{\mathrm{O}_{z}}$ and $C_{z}$ are obtained by equation (11), $B_{z}(t)=T_{2}^{-1} B_{o}(t)$. Details on how to construct $T_{2}$ and the conditions for existence is described in [18]. Assume relative degree $p_{i}$ for each of the $m$ subsystems. Then

$$
B_{z}(t)=\operatorname{diag}\left\{\left[\begin{array}{llll}
0 & \cdots & 0 & 1
\end{array}\right]_{1 \times p_{i}}^{T}\right\}_{n \times m}
$$

Apply the Lyapunov coordinate transformation $z=T_{2}(t) \tilde{\xi}$ to the closed-loop system under state feedback, equation (16), and obtain

$$
\begin{aligned}
& \dot{z}=F\left(T_{1}(t) z, \tilde{\mu}, t, \bar{\xi}(t), \bar{\mu}(t)\right) \\
& \tilde{\eta}=H\left(T_{1}(t) z, \tilde{\mu}, t, \bar{\xi}(t), \bar{\mu}(t)\right)
\end{aligned}
$$

Thus the state feedback law (15) is now 


$$
\tilde{\mu}=K_{z} z
$$

and, the closed-loop system under state feedback in the transformed coordinate is

$$
\begin{aligned}
& \dot{z}=F_{z}\left(z, K_{z} z, t, \bar{\xi}(t), \bar{\mu}(t)\right) \\
& \tilde{\eta}=H_{z}\left(z, K_{z} z, t, \bar{\xi}(t), \bar{\mu}(t)\right)
\end{aligned}
$$

where $K_{z}=K T_{2}^{-1}$ and $F_{z}(\cdot)$ and $H_{z}(\cdot)$ are obtained from $F(\cdot)$ and $H(\cdot)$ accordingly. Note that since system (16) is exponentially stable and Lyapunov transformation preserves the stability for the transformed system, system (26) is still exponentially stable.

Also apply the Lyapunov transformation $\widehat{z}=T_{2}(t) \widehat{\tilde{\xi}}$ to the observer dynamics (18), and obtain

$$
\begin{aligned}
& \dot{\hat{z}}=F_{z}(\widehat{z}, \tilde{\mu}(t), \bar{\xi}(t), \bar{\mu}(t), t)+K_{o_{H}}(\cdot)(\widehat{\widetilde{\eta}}-\tilde{\eta}) \\
& \widehat{\tilde{\eta}}=H_{z}(\widehat{z}, \tilde{\mu}(t), \bar{\xi}(t), \bar{\mu}(t))
\end{aligned}
$$

Accordingly, the output feedback control law (17) is now

$$
\tilde{\mu}=K_{z} \widehat{z}
$$

Note that $\tilde{z}=\widehat{z}-z$.

The following theorem extends time-invariant high-gain theory in [2] (Theorem 14.6 in [12]) to time-varying case. The theorem shows that the output feedback controller recovers the performance of the state feedback controller for sufficiently small $\epsilon$, or equivalently, sufficiently high observer bandwidth, or observer gain.

Theorem Consider the closed-loop system of the plant (24) and the output feedback controller (27) \& (28). Suppose the origin of (26) is exponentially stable and $\mathcal{R}$ is the region of attraction. Let $\mathcal{S}$ be any compact set in the interior of $\mathcal{R}$ and $\mathcal{Q}$ be any compact subset of $R^{p}$. Then,

(i) there exists $\epsilon_{1}^{*}>0$ such that, for every $0<\epsilon<\epsilon_{1}^{*}$, the solutions $(z(t), \widehat{z}(t))$ of the closed-loop system, starting in $\mathcal{S} \times \mathcal{Q}$, are bounded for all $t \geq 0$.

(ii) given any $\sigma>0$, there exists $\epsilon_{2}^{*}>0$ and $T_{2}>0$, both dependent on $\sigma$, such that, for every $0<\epsilon<\epsilon_{2}^{*}$, the solution of the closed-loop system, starting in $\mathcal{S} \times \mathcal{Q}$, satisfies

$\|z(t)\| \leq \sigma \quad$ and $\quad\|\widehat{z}(t)\| \leq \sigma, \quad \forall t \geq T_{2} \quad$ (29)

(iii) given any $\sigma>0$, there exists $\epsilon_{3}^{*}>0$ dependent on $\sigma$, such that, for every $0<\epsilon<\epsilon_{3}^{*}$, the solution of the closed-loop system, starting in $\mathcal{S} \times \mathcal{Q}$, satisfying

$$
\left\|z(t)-z_{r}(t)\right\| \leq \sigma, \quad \forall t \geq 0
$$

where $z_{r}(t)$ is the solution of (26), starting at $z(0)$.

(iv) there exists $\epsilon_{4}^{*}>0$ such that, for every $0<\epsilon<\epsilon_{4}^{*}$, the origin of the closed-loop system is exponentially stable and $\mathcal{S} \times \mathcal{Q}$ is a subset of its region of attraction.

The theorem contains four parts. In Part (i) the boundedness of the closed-loop state variables is established. Part (ii) asserts that the bound of the solutions can be made arbitrarily small in the steady state by increasing the observer bandwidth. Part (iii) establishes the recovery of the performance under state feedback using the high-bandwidth (high-gain) observer. Part (iv) assures the recovery of the exponential stability.

Due to page limit, detailed proof of the Theorem is omitted here, which can be found in [18]. Here we outline the key steps of the proof, including an enabling lemma. The proof utilizes singular perturbation theory. Let

$$
\chi_{i j}=\frac{\widehat{z}_{i j}-z_{i j}}{\epsilon^{p_{i}-j}}=\frac{\tilde{z}_{i j}}{\epsilon^{p_{i}-j}}
$$

$1 \leq i \leq m$ and $1 \leq j \leq p_{i}$ be the scaled observer error. Hence, we have $\widehat{z}=z+D(\epsilon) \chi$, where

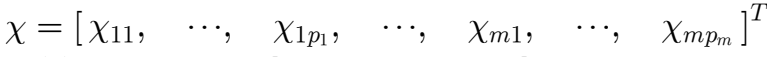

$$
\begin{aligned}
& D(\epsilon)=\operatorname{block} \operatorname{diag}\left[D_{1}, \quad \cdots, \quad D_{m}\right] \\
& D_{i}=\operatorname{diag}\left[\epsilon^{p_{i}-1}, \quad \cdots, \quad 1\right]_{p_{i} \times p_{i}}
\end{aligned}
$$

Then, the dynamics of the closed-loop system is

$$
\begin{aligned}
& \dot{z}=F_{z}\left(z, K_{z}(z+D(\epsilon) \chi), t, \bar{\xi}(t), \bar{\mu}(t)\right) \\
& \tilde{\eta}=H_{z}\left(z, K_{z}(z+D(\epsilon) \chi), t, \bar{\xi}(t), \bar{\mu}(t)\right) \\
& \epsilon \dot{\chi}=A_{0}(t) \chi+\epsilon \Delta_{b}
\end{aligned}
$$

where $\Delta_{b}=D^{-1}(\epsilon) B_{z} \Delta=B_{z} \Delta$, due to the special forms of $B_{z}$ and $D(\epsilon)$ and $(1 / \epsilon) A_{0}(t)=D^{-1}(\epsilon)\left(A_{z}(t)+\right.$ $\left.K_{o_{z}}(t) C_{z}(t)\right) D(\epsilon)$. Let $V(z)$ and $W(\chi)$ be the Lyapunov functions of the slow and fast model, respectively. Define the sets $\Omega_{c}$ and $\Sigma$ by $\Omega_{c}=\{V(z) \leq c\}$ and $\Sigma=\{W(\chi) \leq$ $\left.\varrho \epsilon^{2}\right\}$. The first step of the proof for Part (i) is accomplished by showing that for sufficiently large $\varrho$, there exists an $\epsilon_{1}$, such that for all $0<\epsilon \leq \epsilon_{1}$, the origin of the closed-loop system is exponentially stable and the set $\Omega_{c} \times \Sigma$ is a positively invariant subset of the region of attraction. The second step shows there exists an $\epsilon_{2}$, such that for all $0<\epsilon \leq \epsilon_{2}$, the trajectory enters the set $\Omega_{c} \times \Sigma$ in finite time. Part (ii) is also proved in two steps. The first step shows that we can find an $\epsilon_{3}=\epsilon_{3}(\sigma) \leq \epsilon_{1}^{*}$, such that for every $0<\epsilon \leq \epsilon_{3}$, $\|\chi(t)\| \leq \sigma / 2, \forall t \geq T\left(\epsilon_{3}\right)=\tilde{T}(\sigma)$. Then it is shown that we can choose $\epsilon_{4}=\epsilon_{4}(\sigma) \leq \epsilon_{1}^{*}$, such that for all $0<\epsilon \leq \epsilon_{4}$, $\|z(t)\| \leq \sigma / 2, \forall t \geq \tilde{T}$. Then $\|\widehat{z}(t)\| \leq \sigma / 2$, since $\widehat{z}=z-$ $D(\epsilon) \chi$, and $\|D(\epsilon)\|=1$. Part (iii) is proved by dividing the interval $[0, \infty)$ into three intervals $[0, T(\epsilon)),\left[T(\epsilon), T_{3}\right)$ and $\left[T_{3}, \infty\right)$, and showing (30) for each interval. The proof of the last part uses the fact that (26) is exponentially stable. A Lyapunov function of the overall closed-loop system is constructed based on the Lyapunov function of (26), and is shown to satisfy the exponential stability condition.

The proof also relies on (Theorem 7.4, p117, [23]), (Theorem 7.8, p120, [23]) and (Theorem 4.14, p102, [12]) to guarantee the existence of Lyapunov function for the reduced system and the boundary layer system, and the following Lemma that facilitates the proof of the standard singular perturbation problem by time-scale separation for time-varying dynamics. From the synthesis formula in [15][16], the coefficients of the LPDO associated with the observability canonical form contain $\omega(t)$ and the derivatives of $\omega(t)$. Let $\tau=t / \epsilon$, then $d \omega(t) / d t=$ $(1 / \epsilon)(d \omega(\epsilon \tau) / d \tau)$ and $A(t, d t)=A(\epsilon \tau,(1 / \epsilon) d \tau)$. The following Lemma relates the high gain TLO with the reference TLO through time-scaling.

Lemma Suppose the PD-spectrum of a SISO LTV system is represented as $\Upsilon_{n}=\left\{\rho_{k}(t)=\bar{\rho}_{k} \omega(t)\right\}_{k=1}^{n}$. If the PD-spectrum is scaled up by $1 / \epsilon$, such that $\Upsilon_{H_{n}}=\left\{\rho_{H_{k}}(t)=\bar{\rho}_{k} \cdot \omega_{H}(t)\right\}_{k=1}^{n}$, where, $\omega_{H}(t)=\omega(t) / \epsilon$, the observability canonical realizations of $\Upsilon_{n}$ and $\Upsilon_{H_{n}}$, denoted as $A(t)$ and $A_{H}(t)$ respectively, satisfy

$$
(1 / \epsilon) A(\epsilon \tau, d \tau)=D^{-1}(\epsilon) A_{H}(\epsilon \tau, d \tau) D(\epsilon)
$$

where $D(\epsilon)=\operatorname{diag}\left[\epsilon^{n-1}, \cdots, \quad 1\right]_{n \times n}$. 
The proof of the Lemma utilizes PD-modal matrix (21) and synthesis formula [15][13] of LPDO from a PDspectrum. First, a second-order example is presented to illustrate the idea and validity of the Lemma.

Suppose the PD-eigenvalues of a second order LTV system is given by $\rho_{1}(t)=\overline{\rho_{1}} \omega(t), \rho_{2}(t)=\overline{\rho_{2}} \omega(t)$. The observability canonical form realization is given by the synthesis formula [15][13] and LPDO canonical form realization [18] as

where

$$
A(t, d t)=\left[\begin{array}{cc}
0 & 1 \\
-\alpha_{1}(t, d t) & -\alpha_{2}(t, d t)
\end{array}\right]
$$

$$
\begin{aligned}
& \alpha_{1}(t, d t)=\overline{\rho_{1}} \overline{\rho_{2}} \omega^{2}(t) \\
& \alpha_{2}(t, d t)=-\left(\overline{\rho_{1}}+\overline{\rho_{2}}\right) \omega(t)-\frac{\dot{\omega}(t)}{\omega(t)}
\end{aligned}
$$

Now scale up the bandwidth $\omega(t)$ by $\frac{1}{\epsilon}$ and obtain $\omega_{H}(t)$ $=\omega(t) / \epsilon$. Then the observability canonical form becomes

where

$$
A_{H}(t, d t)=\left[\begin{array}{cc}
0 & 1 \\
-\alpha_{1_{H}}(t, d t) & -\alpha_{2_{H}}(t, d t)
\end{array}\right]
$$

$$
\begin{aligned}
& \alpha_{1_{H}}(t, d t)=\overline{\rho_{1}} \overline{\rho_{2}} \omega^{2}(t) / \epsilon^{2} \\
& \alpha_{2_{H}}(t, d t)=-\left(\overline{\rho_{1}}+\overline{\rho_{2}}\right) \omega(t) / \epsilon-\frac{\dot{\omega}(t)}{\omega(t)}
\end{aligned}
$$

Let $\tau=t / \epsilon$ and notice that $d \omega(t) / d t=(1 / \epsilon)(d \omega(\epsilon \tau) / d \tau)$, then

thus

$$
A_{H}(\epsilon \tau, d \tau)=\left[\begin{array}{cc}
0 & 1 \\
-\alpha_{1}(\epsilon \tau, d \tau) / \epsilon^{2} & -\alpha_{2}(\epsilon \tau, d \tau) / \epsilon
\end{array}\right]
$$

$$
D^{-1}(\epsilon) A_{H}(\epsilon \tau, d \tau) D(\epsilon)=\frac{1}{\epsilon} A(\epsilon \tau, d \tau)
$$

\section{Proof of the Lemma}

As defined in [15][13], the coefficients $\alpha_{k}(t)$ of the companion form realization can be synthesized from the PDspectrum $\left\{\rho_{i}(t)\right\}_{i=1}^{n}$ by

$$
\alpha_{k}(t)=\frac{\tilde{v}_{k, n+1}(t)}{\operatorname{det} V_{n}\left(\rho_{1}(t), \cdots, \rho_{n}(t)\right)}
$$

With the assumption in the previous section that $\Upsilon_{i}=\left\{\rho_{k}(t)=\bar{\rho}_{k} \omega(t)\right\}_{k=1}^{n}$, we have

$$
\operatorname{det} V_{n}(\omega(t))=\operatorname{det} \widehat{V}_{n}(\omega(t)) \operatorname{det} \bar{V}_{n}=\omega^{\frac{n(n-1)}{2}}(t) \prod_{1 \leq i<j \leq n}\left(\bar{\rho}_{j}-\bar{\rho}_{i}\right)
$$

And $\tilde{v}_{i j}(t)$ denotes the algebraic cofactor of $v_{i j}(t)$ in the $(n+1) \times(n+1)$ matrix

$$
\begin{aligned}
& V_{n+1}\left(\rho_{1}, \cdots, \rho_{n}, \rho\right) \\
& =\left[v_{i j}\right]=\left[\begin{array}{ccc|c} 
& & & \\
& V_{n}\left(\rho_{1}, \cdots, \rho_{n}\right) & & \\
& & \mathcal{D}_{\rho}\{1\} \\
\vdots \\
\mathcal{D}_{\rho_{1}}^{n}\{1\} & \cdots & \mathcal{D}_{\rho_{n}}^{n}\{1\} & \mathcal{D}_{\rho}^{n}\{1\}
\end{array}\right]
\end{aligned}
$$

With the time-varying bandwidth being scaled up by $1 / \epsilon$, i.e. $\omega_{H}(t)=\omega(t) / \epsilon$, we now proceed to show that the coefficients $\alpha_{k}(t)$ are scaled up by $\epsilon^{-(n+1-k)}$.

The determinant of the PD-modal matrix is given by

$$
\begin{aligned}
\operatorname{det} V_{n}\left(\omega_{H}(t)\right) & =(1 / \epsilon)^{\frac{n(n-1)}{2}}\left(\omega^{\frac{n(n-1)}{2}}(t) \prod_{1 \leq i<j \leq n}\left(\bar{\rho}_{j}-\bar{\rho}_{i}\right)\right) \\
& =(1 / \epsilon)^{\frac{n(n-1)}{2}} \operatorname{det} V_{n}(\omega(t))
\end{aligned}
$$

Now, we look into the numerator of the synthesis formula of $\alpha_{k}(t)$. We want to show that

$$
\widehat{v}_{i j}\left(\omega_{H}(t), d t\right)=\frac{1}{\epsilon^{i-1}} \widehat{v}_{i j}(\omega(\epsilon \tau), d \tau)
$$

using mathematical induction on $i$. For $i=1$, $\widehat{v}_{11}\left(\omega_{H}(t), d t\right)=1$ and $\widehat{v}_{11}(\omega(\epsilon \tau), d \tau)=1$, thus $\widehat{v}_{11}\left(\omega_{H}(t), d t\right)$ $=\frac{1}{\epsilon^{1-1}} \widehat{v}_{11}(\omega(\epsilon \tau), d \tau)$, and Equation (32) holds for $i=1$. Now assume Equation (32) holds for $i=k-1$. For $i=k$,

$$
\begin{aligned}
\widehat{v}_{i j}\left(\omega_{H}(t), d t\right)= & \dot{\widehat{v}}_{i-1, j}\left(\omega_{H}(t), d t\right)+\omega_{H}(t) \widehat{v}_{i-1, j-1}\left(\omega_{H}(t), d t\right) \\
= & \frac{1}{\epsilon^{i-2}} \frac{d\left(\widehat{v}_{i-1, j}(\omega(\epsilon \tau), d \tau)\right)}{d t} \\
& +\frac{1}{\epsilon} \frac{1}{\epsilon^{i-2}} \omega(\epsilon \tau) \widehat{v}_{i-1, j-1}(\omega(\epsilon \tau), d \tau) \\
= & \frac{1}{\epsilon^{i-1}} \widehat{v}_{i j}(\omega(\epsilon \tau), d \tau), \quad \forall j \leq k
\end{aligned}
$$

Thus

$$
\widehat{V}_{n+1}\left(\omega_{H}(t), d t\right)=\operatorname{diag}\left(\left[1, \epsilon^{-1}, \cdots, \epsilon^{-n}\right]\right) \widehat{V}_{n+1}(\omega(\epsilon \tau), d \tau)
$$

and

$$
\tilde{v}_{k, n+1}\left(\omega_{H}(t), d t\right)=\prod_{i=0}^{n}(1 / \epsilon)^{i} \epsilon^{k-1} \tilde{v}_{k, n+1}(\omega(\epsilon \tau), d \tau)
$$

Therefore

$$
\alpha_{k}\left(\omega_{H}(t), d t\right)=\frac{\tilde{v}_{k, n+1}\left(\omega_{H}(t), d t\right)}{\operatorname{det} V_{n}\left(\omega_{H}(t)\right)}=\frac{1}{\epsilon^{n-k+1}} \alpha_{k}(\omega(\epsilon \tau), d \tau)
$$

With the above relationship established, (31) is ready verified by direct computation.

Remark 1. The theorem is stated and proved in the transformed coordinate. However, due to the nature of the Lyapunov transformation, i.e. the boundedness of $T_{1}, T_{1}^{-1}$ and $\dot{T}_{1}$, the theorem still hold in the original coordinate for $x, \widehat{x}$ and $\tilde{x}$ with different $\epsilon_{i}^{*}, i=1, \cdots, 4$.

Remark 2. According to the theorem, the tracking performance should be improved with the decrease of $\epsilon$. However, the system output may exhibit an impulse-like behavior, which is called peaking phenomenon ([12], p613), when $\epsilon$ is sufficiently small. The peaking phenomenon can be overcome by saturating the control to create a buffer that protects the plant from peaking, which, in effect, reduces the observer gain of the output feedback system during peaking. Thus a TVB TLO can be used to handle peaking as well.

Remark 3. Theoretically speaking, reducing $\epsilon$ will enhance the steady state tracking performance. However, besides the peaking phenomenon, the measurement noise and unmodeled high-frequency sensor dynamics also put a practical limit on how small $\epsilon$ could be, which should be considered in the tuning process.

Remark 4. Expanding the bandwidth of the observer dynamics by reducing $\epsilon$ results in faster response of the observer dynamics, yielding quicker decay rate of vanishing perturbation $\delta_{v}(\cdot)$ in (19). However, from the proof, one can see that the main purpose of the high-gain observer is to deal with the non-vanishing perturbation and to reduce the steady-state error cause by non-vanishing perturbation.

\section{NUMERICAL EXAMPLE}

A numerical example of the proposed TLO for an unstable, nonminimum phase, stiff nonlinear benchmark model is presented in [18]. A simulation comparison study of the proposed TLO with EKF based on the same model is also presented in [18]. 
A MIMO high-gain TLO design example with hardwarein-loop simulation test for a 3 Degrees-of-Freedom (3DOF) differential thrust control testbed named "Quanser UFO" is presented in [17].

\section{CONCLUSION}

In this paper, a time-varying high gain trajectory linearization observer for nonlinear system is presented. Some remaining problems that were not solved or even addressed in the previous TLO paper [13] are dealt with in this paper, such as the robustness of the proposed observer design method to modeling errors, and the application to MIMO nonlinear systems. The main result has shown that, using high-gain (bandwidth), the TLO based output feedback recovers the state feedback performance, even for timevarying observer gains (bandwidth). Moreover, the timevarying high gain TLO relaxes the restriction of existing time-invariant high gain observer to affine nonlinear systems to allow any linearizable nonlinear system. Real-time hardware-in-the-loop test using the proposed design on a $3 \mathrm{DOF}$ flight control testbed is presented in [17], and a simulation comparison study is given in [18]. Detailed proof of the main theorem in this paper and more design case studies are included in [18].

\section{REFERENCE}

[1] E. A. Misawa and J. K. Hedrick, "Nonlinear observers: A state-of-theart survey," Trans. of ASME J. of Dyn. Sys., Measurement and Ctrl., vol. 111, pp. 344-352, 1989.

[2] H. Nijmeijer and T. I. Fossen, (editors), New Direction in Nonlinear Observer Design, Vol. 244 of Lecture Notes in Control and Information Sciences, Springer, London, 249-268, 1999.

[3] A. J. Krener and A. Isidori,"Linearization by output injection and nonlinear observer," System \& Control Letter, vol. 3, pp. 47-52, 1983.

[4] M.Q. Xiao, "The global existence of nonlinear observer with linear error dynamics: A topological point of view," Systems \& Control Letters, vol. 55, 849-858, 2006.

[5] C. Kravaris, V. Sotiropoulos, C. Georgiou, N. Kazantzis, M. Xiao, A.J. Krener, "Nonlinear observer design for state and disturbance estimation," Systems Control Letters, vol. 56 730-735, 2007.

[6] M. S. Grewal and A. P. Andrews, Kalman filtering, Prentice Hall, Englewood Cliffs, NJ, 1993.

[7] P. S. Maybec, Stochastic models, estimation and control, Academic Press, New York, 1982.

[8] W. T. Baumann and W. J. Rugh,"Feedback control of nonlinear systems by extended linearization," IEEE Trans. on Automatic Control, vol. 31, pp. 40-46, 1986.

[9] A.J. Krener, "The convergence of the extended Kalman filter," Directions in mathematical systems theory and optimization," 173182, Lect. Notes in Ctrl. \& Inform. Sci., 286, Springer, Berlin, 2003.

[10] A.N. Atassi and H.K. Khalil, "A separation principle for the stabilization of a class of nonlinear systems," IEEE Trans. Automatic Control, 44:1672-1687, 1999.

[11] F. Esfandiari and H.K. Khalil, "Output feedback stabilization of fully linearizable systems," Int. J. Control, 56:1007-1037, 1992.

[12] H. K. Khalil, Nonlinear systems, 3 ed, Prentice hall, NJ, 2002.

[13] R. Huang, M. C. Mickle and J. Zhu,"Nonlinear Time-varying Observer Design Using Trajectory Linearization," Proceedings of ACC, Denver, Colorado, pp. 4772-4778, June, 2003.

[14] J. J. Zhu,"A necessary and sufficient stability criterion for linear timevarying systems," Proc. of 28th IEEE SSST, pp. 115-129, 1996.

[15] J. J. Zhu,"PD-spectral theory for multivariable linear time-varying systems," Proc., 36th IEEE CDC, San Diego, CA, 3908-3913, 1997.
[16] J. J. Zhu and M. C. Mickle,"Synthesis of time-varying bandwidth filters based on all-pole LTI prototypes," Proc. of ACC, Philadelphia, PA, pp. 2889-2894, 1998.

[17] R. Huang, and J. Zhu, "MIMO High Gain Trajectory Linearization Observer Design and Hardware-in-the-loop Test," Proc. of ACC, New York, pp. 248-253, July, 2007.

[18] R. Huang, "Output feedback tracking control of nonlinear time-varying systems by trajectory linearization," Ph. D. dissertation, the School of EECS, Ohio University, Athens, Ohio, 2007

[19] M. C. Mickle, R. Huang and J. J. Zhu, "Unstable, nonminimum phase, nonlinear tracking by trajectory linearization control," Proceedings, IEEE Conference on Ctrl. App., Taibei, Taiwan, pp. 812-818, 2004.

[20] S. Limanond and K. S. Tsakalis,"Adaptive and non-adaptive 'poleplacement' control of multivariable linear time-varying plants," Int $J$ Control, vol. 74, no. 5, pp. 507-523, 2001.

[21] L. Markus and M. Yamabe,"Global stability criteria for differential systems,” Osaka Math. J., vol. 12, pp. 305-317, 1960.

[22] M.Y. Wu, “A note on stability of linear time-varying systems," IEEE Trans. on Automatic Control, vol. 19, pp. 162, 1974

[23] Wilson J. Rugh, Linear system theory, 2 ed, Prentice-Hall, Inc., Upper Saddle River, NJ, 1996.

[24] L. M. Silverman, "Transformation of time-variable systems to canonical (phase-variable) form," IEEE Trans. on Auto. Control, vol. 11 , pp. 300-303, 1966.

[25] D. Bestle and M. Zeitiz,"Canonical form observer design for nonlinear time-variable systems," International J. of Ctrl, vol. 38, pp. 419-431, 1983.

[26] W. A. Wolovich, "On the stabilization of controllable systems," IEEE Trans. on Automatic Control, vol. 13, pp. 569-572, 1968.

[27] C. E. Seal and A. R. Stubberud,"Canonical forms for multiple-input time-variable systems," IEEE Trans. on Automatic Control, vol. 6, pp. 704-707, 1969 . 